premenopausal and postmenopausal women. It is not true that most adjuvant trials show a clear difference between premenopausal and postmenopausal patients. With a few exceptions, ${ }^{3}{ }^{4}$ for which plausible explanations might be proposed, relapse free survival and survival were not differently affected by chemotherapy in premenopausal and postmenopausal women. Oestrogen receptor state and drug induced amenorrhoea do not affect the response rate to adjuvant chemotherapy. ${ }^{5}$ Data on relative toxicity of the 24 hour approach, which I advocate, compared with more standard regimens clearly show an appreciable reduction in toxicity without loss of therapeutic effect at least in advanced disease. It is not true that these comparisons have not been published; they are contained in the references appended to my original letter (24 September, p 908). As Dr De Vita, director of the National Cancer Institute in Washington has pointed out, "The worst problem is pessimism."

I think that Dr Smith has concentrated too much on the admittedly disappointing results of early studies. Your readers are surely entitled to know that at least nine studies have now shown unequivocally that the clinical course of breast cancer can be favourably altered for the first time for several decades. ${ }^{5}$ (A Buzdar and others, Meeting of American Society of Clinical Oncologists 1983; L Israel and others, 13th International Congress of Chemotherapy, 1983.) Although the best adjuvant protocol is not yet known, studies are currently being planned in Europe and the United States to see if the present encouraging trends can be improved. I think it is much better to cooperate in these future studies than continually to draw sweeping conclusions from the relative failure of past studies. Meanwhile I think any disagreements between Dr Smith and myself should be judged on the basis of the evidence provided in the references.

L A PRICE

International Advisory Council,

New York Chemotherapy Foundation

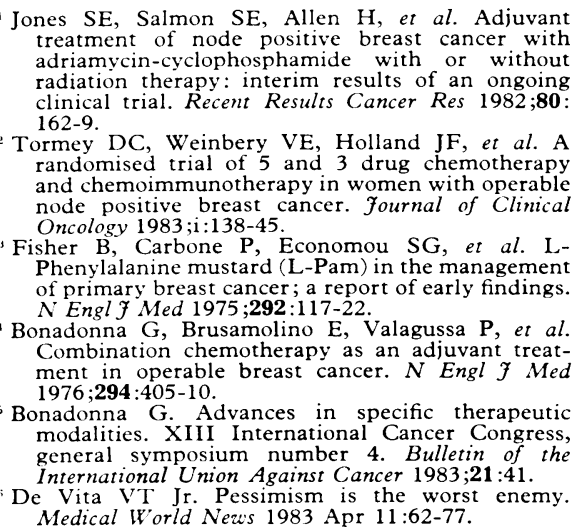

\section{New criteria and classification for diabetes mellitus}

SIR,-We wish to comment on a possible anomaly that appears to exist in the standard conditions used in the glucose tolerance test, restated by the British Diabetic Association officers (20 August, p 560).

The recommended load is $75 \mathrm{~g}$ oral glucose, obtained from our pharmacy in $75 \mathrm{~g}$ portions of D-glucose monohydrate (molecular weight 198) (pharmaceutical name, dextrose). This amount would be equivalent to about $68 \mathrm{~g}$ of anhydrous D-glucose (molecular weight 180) (biochemical name, glucose). At least one commercially prepacked product for use in glucose tolerance tests states on the label that the contents are $75 \mathrm{~g}$ glucose, while the package insert states that the contents are $75 \mathrm{~g}$ glucose monohydrate.

There are, therefore, two ways of interpreting the required glucose load, either by giving anhydrous D-glucose $(75 \mathrm{~g})$ or Dglucose monohydrate $(75 \mathrm{~g})$, and this depends on the difference between the pharmaceutical and biochemical definition, because of the use of imprecise nomenclature. We appreciate that we may be seen to be rather pedantic in making this comment, as the two different glucose loads are unlikely to affect appreciably the nature of the glucose tolerance curves. It is also probable that most biochemistry departments obtain glucose preparations from their hospital pharmacy or by using commercially prepacked preparations, so that a discrepancy may not exist.

If standard diagnostic values are clearly stated for the interpretation of glucose tolerance tests then standard conditions for conducting the tesi should be applied, and the $75 \mathrm{~g}$ oral glucose load should be a consistent quantity. Directions for conducting the test such as "75 g glucose (dextrose) orally" could eliminate possible misinterpretation.

E J HINDLE G M Rostron

Biochemistry Department,

The District Laboratory,

Preston Infirmary,

SIR,-The World Health Organisation's recommendations referred to in the letter from Professor Keen and his colleagues which include the statement: "Fasting values below $6 \mathrm{mmol} / 1(198 \mathrm{mg} / 100 \mathrm{ml})$ exclude the diagnosis of diabetes" appear in subsection 2.2.3 "Proposed diagnostic procedure and criteria") of the WHO report. In subsection 2.2.1 ("General considerations") of the same report, however, appears the statement: "The evidence suggests that a diagnosis of diabetes is justifiable even when fasting glucose levels are normal."

Surely these two statements are contradictory and will cause confusion?

\section{Biochemistry Department,
Airedale General Hospital, \\ Airedale
Steeton, \\ Steeton, \\ Weighley,}

C SANDERSON

\section{Twelfth rib syndrome}

SIR,-I was astonished to read of the four case reports of rib resection (27 August, p 586) for the relief of dorsal nerve root pain.

The condition may well have been named slipping rib, clicking rib, or rib syndrome, but it is better known as intercostal nerve neuralgia. I have called it dorsatica, ${ }^{1}$ and it may mimic renal pain, appendicitis, cholecystitis, pleurisy, etc, depending on the root affected. The most likely cause is nerve root compression-that is, prolapsed disc. Spinal manipulation usually relieves it, as does nerve root block using local anaesthetic and steroids. Rarely the pain may be referred from the costovertebral joint, when the same injection into the joint space relieves the pain.

I would humbly suggest that the authors refer the other cases to the local pain relief clinic, as I do not think that rib resection is a simple surgical procedure, let alone a pretty one.

F F CASALE

Pain Relief Clinic,

Essex County Hospital,

Colchester,

Essex ' Casale FF. A proposed simple classification of
musculo-skeletal pain. Ann R Coll Surg Engl (in press)

${ }_{*}^{*}$ We sent a copy of this letter to the authors, who reply below.-ED, $B M$ F.

SIR,-Dr Casale takes issue with us in respect of the name, aetiology, and treatment of a condition which we believe constitutes one differential diagnosis of loin pain and which we choose to call "twelfth rib syndrome." We feel that the title is apt for this specific condition because it indicates the site of pain and its aetiology, suggests an approach to treatment, and, hopefully, may lead to an increase in clinical awareness of its existence as a distinct entity.

We have made it clear in our paper that the particular syndrome we were discussing constituted a severe intercostal neuralgia, related to the 11 th or 12 th rib, or both. The pain could be exactly reproduced by manipulating, and easily cured by simple resection of, the relevant rib or ribs. Neither of these criteria would, of course, apply to pain of spinal origin.

Our patients had all suffered prolonged and severe intercostal neuralgia associated with their 11th or 12th ribs, and we have no reservations about offering such unfortunate individuals a simple surgical procedure to help them, once the specific diagnosis has been accurately made.

We have tried the therapeutic use of steroids and local anaesthetic agents but have to report that we have been unable to achieve relief of pain in our patients for longer than the duration of action of the local anaesthetic agent and so we have abandoned the technique in this condition.

We trust these further comments on this syndrome have clarified the entity for $\mathrm{Dr}$ Casale but should he require any further information to enable him to include it in his "Proposed simple classification of musculoskeletal pain" we would be happy to correspond with him direct.

Derek G Machin

Roval Liverpool Hospital Liverpool L7 8XP

Arrowe Park Hospital,

Wirral

Merseyside 4995

JOHN M SHENNAN

\section{Anorexia nervosa}

SIR,-In his competent review of anorexia nervosa (24 September, p 855) Professor A H Crisp outlines the various theories of aetiology without mentioning the role of the hypothalamus.

It is plain that there is a strong link in development at puberty between the switching 
on of the sexual homoeostat and certain prior energy requirements. Sexual maturity does not begin until a certain critical body weight is reached, and this is indicated in the diagram provided by Professor Crisp. In fact, the earlier age at which puberty occurs at the present time must be related to improved quantities and distribution of food supplies It is also clear from the diagram of the typical evolution of anorexia nervosa that the phobic avoidance of mature body weight produces a reduction below the $50 \mathrm{~kg}$ weight level at which this phase of development normally commences. Critical weight, and weight gain from the age of 9 is a preparation for a period of rapid cell growth, and the storage of cholesterol is necessary since this substance is a unique and important constituent of the plasma membrane of mammalian cells. A cell will not enter the mitotic cycle unless the cholesterol level required for development of the membranes of daughter cells is attained.'

Means for the integration and coordination of the functions of separate endocrine glands are provided at the pituitary level of the endocrine system, but interference with energy intake will reverse this normal process and retard aging. It is interesting to note that it has often been reported that the same negative emotions increase appetite and body weight in middle aged persons. ${ }^{2}$

Croydon CRO 7HL

M KeITh Thompson

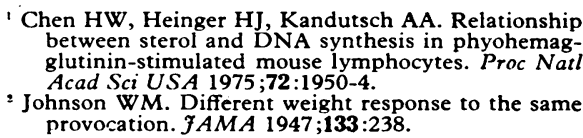

\section{Epileptic seizures in general practice}

SIR,-The findings of Dr D M G Goodridge and Dr S D Shorvon (3 September, p 641) may come as no surprise to general practitioners but will be valuable to hospital physicians in putting epilepsy into perspective. The results would have been more convincing, however, if the authors had described precisely how they axcluded anoxic seizures ${ }^{1}$ (nonepileptic convulsive syncope) and indicated whether or not they included anoxic-epileptic seizures" as "epilepsy." They excluded febrile convulsions (only in those aged between 12 months and 5 years, for reasons unexplained) but included fits after alcohol withdrawal; surely these fits comprise both "stress convulsions," genetically linked to febrile convulsions, ${ }^{3}$ and vagal convulsive syncope.

I do not think that it is sufficient for the diagnosis of epilepsy that it has been considered certain by a neurologist or a paediatrician. I have no reason to believe that our neurologists and paediatricians are less diagnostically alert than their southern counterparts, but nevertheless of 41 patients with reflex cardiovagal convulsive syncope recently studied $40 \%$ had been diagnosed as having epilepsy, $9 \%$ with enough confidence for each to be prescribed a mean of 1.8 antiepileptic drugs (unpublished data). The diagnosis was missed because, having excluded breath holding attacks or vasovagal syncope, sufficient attention was not paid to the provocation, to the heart stopping precipitating factors ${ }^{4}$-head bump, pain, fright, or surprise.

On being introduced to the ways of the vagus and the concept of reflex asystole, patients and parents ask "Why don't the doctors know about this ?" Those who write about the demography of epilepsy should make it crystal clear that they are cognoscenti and know the sheep from the goats.

\section{J B P STEPHENSON}

Fraser of Allander Unit,

Royal Hospital for Sick Children,

Yorkhill

Glasgow G3 8SJ

'Stephenson JBP. Reflex anoxic seizures and ocular compression. Dev Med Child Neurol 1980;22: 380-6.

Stephenson JBP. Febrile convulsions and reflex anoxic seizures. In: Rose FC, ed. Research progress in epilepsy. London: Pitman, 1983:244.

Friis ML, Lund M. Stress convulsions. Arch Neurol $1974 ; 31: 155-9$.

Braham J, Hertzeanu H, Yahini JH, Neufeld HN. Reflex cardiac arrest presenting as epilepsy. Ann Neurol $1981 ; 10: 277-8$

** We sent a copy of this letter to the authors, who reply below.-ED, $B M \mathcal{H}$.

SIR,-The problems of diagnosis in our study were discussed in detail, and we quite agree that anoxic seizures may be mistaken for epilepsy and that care should be taken to exclude this. The problem is perhaps greatest among children, and in the paper of Friis and Lund, which Dr Stephenson quotes, "stress convulsions" were identified in only $3 \%$ of 1250 patients with convulsive disorders.

Tonbridge,
Kent

D M G GOODRIDGE

S D SHORVON

National Hospital for Nervous Diseases,

Queen Square,
London WC1N 3BG

\section{Dihydrocodeine overdose treated with naloxone infusion}

SIR,-We would like to make several points in response to Dr Nancy Redfern's lesson of the week (10 September, p 751). Firstly, at least 1.2 to $1.6 \mathrm{mg}$ naloxone is required as an initial dose in severe opiate poisoning. ${ }^{1}$

Secondly, once the trends in the patient described were obviously downwards artificial ventilation should have been started. This would have been preferable to administering further doses of naloxone in an attempt to avoid intermittent positive pressure ventilation, which allowed the patient to reach the brink of respiratory arrest on two occasions. Earlier intermittent positive pressure ventilation would also have corrected the respiratory component of the acidosis, which was undoubtedly aggravating the hyperkalaemia, although reduction of the serum potassium to a safe level would probably still have required dialysis. In our view dialysis should have been considered when the serum potassium had risen to $5 \mathrm{mmol}(\mathrm{mEq}) / \mathrm{l}^{2}$ and certainly started when it was $7.4 \mathrm{mmol} / \mathrm{l}$. The delay allowed a rise to $8.2 \mathrm{mmol} / \mathrm{l}$, which may well have induced cardiac arrest in an already hypoxic myocardium. $^{3}$ (There is no mention of arterial oxygen pressure $\left(\mathrm{PaO}_{2}\right)$ or fractional inspired oxygen $\left(\mathrm{F}_{\mathrm{I}} \mathrm{O}_{2}\right)$ at any point in the text but, with the alveolar gas equation, an arterial carbon dioxide pressure $\left(\mathrm{PaCO}_{2}\right)$ of $14.3 \mathrm{kPa}$ corresponds to a $\mathrm{PaO}_{2}$ of about $4 \mathrm{kPa}$ when breathing room air.) Finally, it is important to note that the manufacturers of naloxone (Du Pont, UK) recommend that the drug be used with caution in the presence of myocardial irritability as ventricular tachycardia may be induced.

In summary, we feel that Dr Redfern was fortunate following her line of management in view of an inadequate sustained response to naloxone. We accept that this treatment has been used with success in other cases of opiate overdosage ${ }^{5}$ but failure is not an indication to soldier on seeking an effective dose; rather it is an indication for intermittent positive pressure ventilation.

I J GORDON

Whiston Hospital,

Prescot,

Merseyside L35 5DR

H L GORDON

Royal Liverpool Hospital,
Liverpool

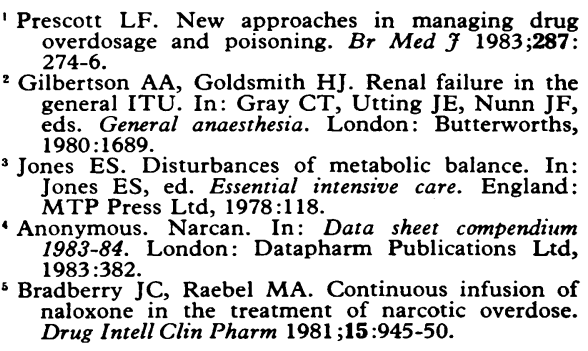

\section{Sickle haemoglobin and pregnancy}

SIR, - We would like to comment on the review by Dr Graham $R$ Serjeant on sickle haemoglobin and pregnancy (3 September, p 628). Women with both sickle cell trait and sickle cell disease should ideally be offered the facilities of genetic counselling and antenatal diagnosis, to which their condition so readily lends itself. Fetoscopy in the second trimester allows globin chain analysis of a fetal blood sample, ${ }^{1}$ while the newer techniques of chorionic biopsy and DNA analysis are applicable in the first trimester. ${ }^{2}$

We feel that it is dangerous to assume that women with sickle cell haemoglobin $\mathrm{C}$ disease (SC) have fewer problems in pregnancy than those with homozygous sickle cell (SS) disease although the unwary clinician may be lulled into a false sense of security by the tendency of women with SC disease to maintain a normal total haemoglobin concentration. ${ }^{3}$ It remains of considerable concern that the prognosis for the fetus in pregnancies complicated by maternal sickle cell disease of all types has improved much less over the past 40 years than that observed for the mother. ${ }^{4}$ Furthermore, despite the relatively low coincidence of pregnancy and sickle cell disease in the United Kingdom four to six maternal deaths from this cause are regularly reported in each triennium. ${ }^{5}$

Our observations at Dulwich Hospital ${ }^{6}$ concur with those reported earlier from Jamaica $^{7}$ in finding no evidence of increased placental infarction to explain the impaired fetal growth and survival common in maternal sickle cell disease. The incidence of maternal red cells showing sickle deformity in the placenta is no greater than that seen in the placentas of women with sickle cell trait (who show no impairment of fetal wellbeing). In any case, few placental vessels are of a calibre that would be obstructed by sickled erythrocytes. We believe that the defective supply line to the fetus is more likely to result from impaired dynamics of the maternal circulation due to increased blood viscosity. 